https://doi.org/10.21516/2072-0076-2019-12-4-8-12

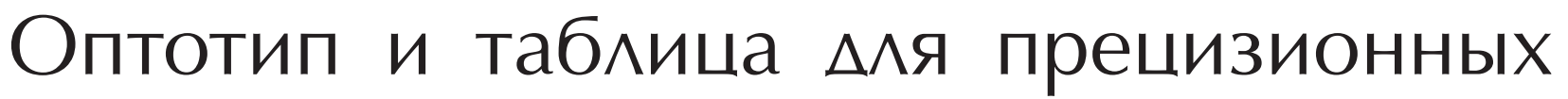 визометрических исследований
}

\author{
А-Г.А. Алиев ${ }^{1}$ А.А-Г. Алиев², М.М. Нурудинов² \\ 1 ГБОУ ВПО «Дагестанский государственный медицинский университет», пл. Ленина, д. 1, \\ г. Махачкала, 367000, Российская Федерация, Республика Дагестан \\ 2 ГБУ НКО «Дагестанский центр микрохирургии глаза», ул. Набережная, д. 12, \\ г. Каспийск, 368300, Российская Федерация, Республика Дагестан
}

Цель работы - разработка и оценка достоверности нового оптотипа и таблицы для прецизионных визометрических исследований. Материал и методы. Обследовано 56 пациентов в возрасте 16-57лет. Критерием включения в исследование явиласьмаксимальная острота зрения (ОЗ) 1,0 и более. ОЗ определяли с использованием трех разных оптотипов: стандартных буквенных оптотипов таблицы Сивиева - Головина, колец Ландольта и предлагаемого нами оптотипа. Результаты. На основе психометрического анализа результатов составлены соответствующие кривые. При использовании нового оптотипа число правильных ответов при ОЗ = 1,0 составило $75 \%$, при ОЗ =1,5-25\%, а при ОЗ =2,0 - $10 \%$, в то время как при использовании колеи, Ландольта и стандартных буквенных оптотипов таблицы Сивцева - Головина получены следуюшие результаты: 1,0$50 \%, 1,5-20 \%, 2,0-10 \%$ и 1,0-45\%, 1,5-15\%, 2,0-5\% соответственно. Заключение. Предложенные оптотип и таблица для визометрических исследований, построенная по принципу логарифмической прогрессии размеров оптотипов, позволяют в значительной мере повысить достоверность оценки ОЗ, а расширенный диапазон величин оптотипов повышает чувствительность методики визометрии.

Ключевые слова: визометрия; таблица для проверки остроты зрения; оптотип

Конфликт интересов: отсутствует.

Прозрачность финансовой деятельности: никто из авторов не имеет финансовой заинтересованности в представленных материалах или методах.

Для цитирования: Алиев А-Г.Д., Алиев А.А-Г., Нурудинов М.М. Оптотип и таблица для прецизионных визометрических исследований. Российский офтальмологический журнал. 2019; 12 (4): 8-12.

doi: 10.21516/2072-0076-2019-12-4-8-12

\section{A new optotype and chart for precision visometry}

Abdul-Gamid D. Aliev¹, Akhmed A.-G. Aliev², Musa M. Nurudinov²

${ }^{1}$ Chair of ophthalmology, Daghestan State Medical University, 1, Ploshchad Lenina, Makhachkala, 367000, Russia

2 Daghestan Center of Eye microsurgery, 12, Naberezhnaya St., Kaspiysk, 368300, Russia musail1988@gmail.com

Purpose. To develop a new optotype and a chart for precision visometric tests and assess their accuracy. Materials and methods. 56 patients aged 16 to 57 years with the maximum visual acuity (VA) of 1.0 or more were examined for VA using three types of optotypes: standard letter optotype based on the Sivtsev - Golovin table, Landolt rings, and a new optotype 
developed by the authors. Results. On the basis of the psychometric analysis, the corresponding curves were drawn. When using the new optotype, the share of correct answers for $V A=1.0$ was $75 \%$, for $V A=1.5-25 \%$, and for $V A=2.0-10$ $\%$. In contrast, Landolt rings and Sivtsev - Golovin optotypes gave the following results, respectively: VA $=1.0-50 \%$, $1.5-20 \%, 2.0-10 \%$ and $V A=1.0-45 \%, V A=1.5-15 \%, V A=2.0-5 \%$. Conclusion. The proposed optotype and the table for visometric tests, based on the principle of logarithmic progression of optotype sizes, significantly increase the accuracy of VA assessment. An extended range of optotype sizes increases the sensitivity of the visometry.

Keywords: visometry; visial acuity; optotype

Conflict of interests: there is no conflict of interests.

Financial disclosure: No author has a financial or property interest in any material or method mentioned.

For citation: Aliev A-G.D., Aliev A.A-G., Nurudinov M.M. A new optotype and chart for precision visometry.

Russian ophthalmological journal. 2019; 12 (4): 8-12 (In Russian). doi: 10.21516/2072-0076-2019-12-4-8-12

Острота зрения (O3) является основным и наиболее значимым параметром, характеризующим состояние зрительного анализатора.

В настоящее время принято выделять три основные ступени, характеризующие центральное зрение: minimum visibile - наименьшее из видимого, minimum separabile способность различить минимальные детали замеченного объекта и minimum cognoscibile - способность опознать объект в целом при наименьших размерах его деталей. Зрительное восприятие зависит не только от угловых размеров объектов, но и от опыта обследуемого и определяется целым рядом других характеристик его высшей нервной деятельности. Простейшим примером оценки О3 в режиме различения служит способность увидеть две точки, находящиеся друг от друга на расстоянии, равном их диаметру, как раздельные. Другим примером может быть способность различить чередующиеся черно-белые полосы минимальной ширины с предельного расстояния [1, 2].

Впервые математическая величина, характеризующая O3, упоминается в 1674 г. в работе астронома R. Hooke, в которой он утверждал, что при нормальном зрении человек способен невооруженным глазом увидеть две звезды, расположенные под углом в 1 мин [2].

Таким образом, у большинства авторов основой для исследования О3 служило определение minimum separabile. С тех пор именно эту величину использовалидля определения О3 [1].

В 1738 г. J. Jurin предпринял попытку исследовать О3 с помощью белых квадратов на черном фоне. Им было отмечено, что ОЗ зависит не только от толщины черты, но и от ее длины [3]. F. Donders впервые предложил использовать в таблицах оптотипы, в которых отношение ширины ножки к высоте всей буквы составляет 1:5. Несмотря на это, ширина букв в его таблицах могла быть произвольной, а очертания букв не во всех отделах имели толщину, равную 1 мин. Он совместно с Н. Snellen предложил известную общую формулу для определения O3, а именно: V=d/D, где $\mathrm{V}$ - O3 (vision acuity), D - расстояние, на котором элементы данного оптотипа видны под углом в 1 мин, а $\mathrm{d}$ - расстояние, на котором удается распознать данный знак [4, 5].

Впервые стандартизованная методика измерения О3 была описана H. Snellen в 1867 г. [6, 7]. Он предложил таблицу (рис. 1), в которой содержались строки прописных букв, оптотипов; размер букв уменьшался от строки к строке в направлении сверху вниз. В качестве оптотипов им были использованы латинские буквы.

В России широкое распространение получила таблица Д.А. Сивцева и С.С. Головина, построен- ная на основе таблицы Снеллена, которая была введена в практику в 1923 г. [8]. Данная таблица помимо буквенных оптотипов включала в себя также и оптотипы Ландольта (рис. 2).

Важным этапом в развитии визометрии явился предложенный в 1888 г. швейцарским офтальмологом E. Landolt оптотип в виде кольца с разрывом (рис. 3). B оптотипе Ландольта соотношение внешнего диаметра кольца к его ширине и к ширине разрыва составляет 5:1:1 [9]. Преимуществом данного оптотипа является то, что при его рассмотрении нивелируется фактор узнаваемости, присущий буквенным и цифровым оптотипам.

При проведении визометрии также крайне важным фактором является сложность контура оптотипов, как, например, буквы, построенные из элементов одинаковой угловой величины, но имеющие различные контуры. Это приводит к тому, что с предельного расстояния такие оптотипы распознаются исследуемым с различным удельным весом ошибочных ответов.

Таким образом, при составлении таблиц для визометрии оптотипы в идеале должны быть изогностичными. Типичными примерами изогностичных оптотипов являются кольца Ландольта, оптотипы Снеллена и Пфлюгера.

В офтальмологической литературе периодически появлялись статьи о создании новых таблиц, однако все они были построены по одному принципу и отличались лишь используемыми шрифтами. Оптотипы в этих таблицах последовательно уменьшались и размер минимальных знаков характеризовал O3, выражавшуюся в угловых градусах или в условных единицах [10]. Работы по созданию новых оптотипов

Рис. 1. Таблица Снеллена для исследования остроты зрения

Fig. 1. Snellen chart for vision acuity measurement

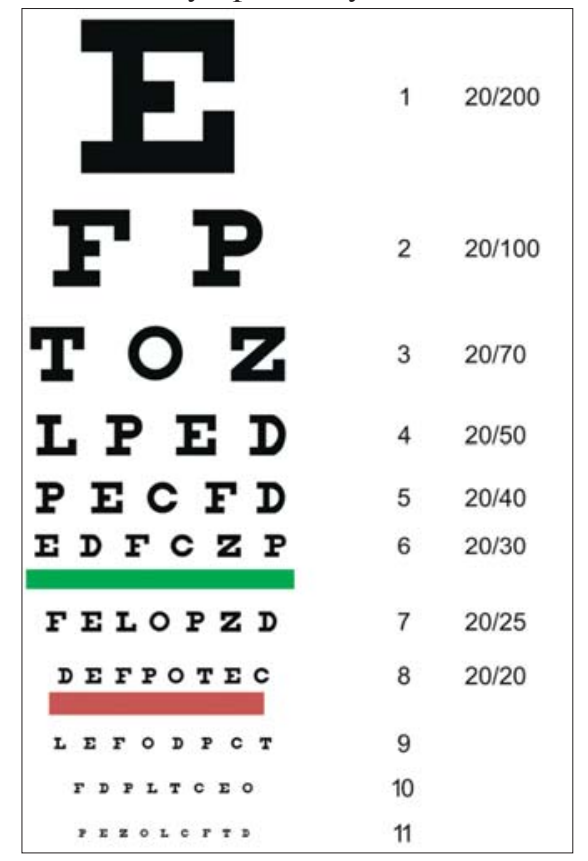

Российский офтальмологический журнал, 2019; 12(4): 8-12 
ведутся и по сей день, что говорит о том, что существующие оптотипы и таблицы для их предъявления все еще далеки от совершенства [11-15].

В частности, широко применяемый и считающийся эталонным оптотип Ландольта имеет ряд недостатков. Так, C. Ferree и G. Rand [16] отметили, что узнавание положения разрыва в кольце до того, как он становится отчетливо видимым, происходит из-за контрастной «неуравновешенности». Для устранения этого эффекта авторами было предложено «уравновешенное» кольцо, толщина которого неравномерна: в области, противоположной разрыву, толщина минимальна.

Схожий по принципу «уравновешивания» оптотип предложили В.Б. Вейнберг и Н.А. Никольская [17] (рис. 4).

K недостаткам оптотипа Ландольта также стоит отнести меридиональную неравномерность детали (разрыва) оптотипа. Данная особенность является физической основой «феномена закругления углов» [1, 16, 18-20] (см. рис. 3). Суть данного феномена заключается в следующем. Разрыв в оптотипе Ландольта представляет собой квадрат со сторонами (a) и (b), при этом его диагональ (c) является гипотенузой равностороннего треугольника и равна a

$\frac{\sin \alpha}{\sin }$ Из этого следует, что диагональный размер разрыва превышает величину его сторон на $\sqrt{2 \mathrm{a}^{2}}-\mathrm{a}$, в процентном соотношении на 29,3\%.

По этой причине расположение разрыва в кольце Ландольта узнается по изменению общего контура объекта

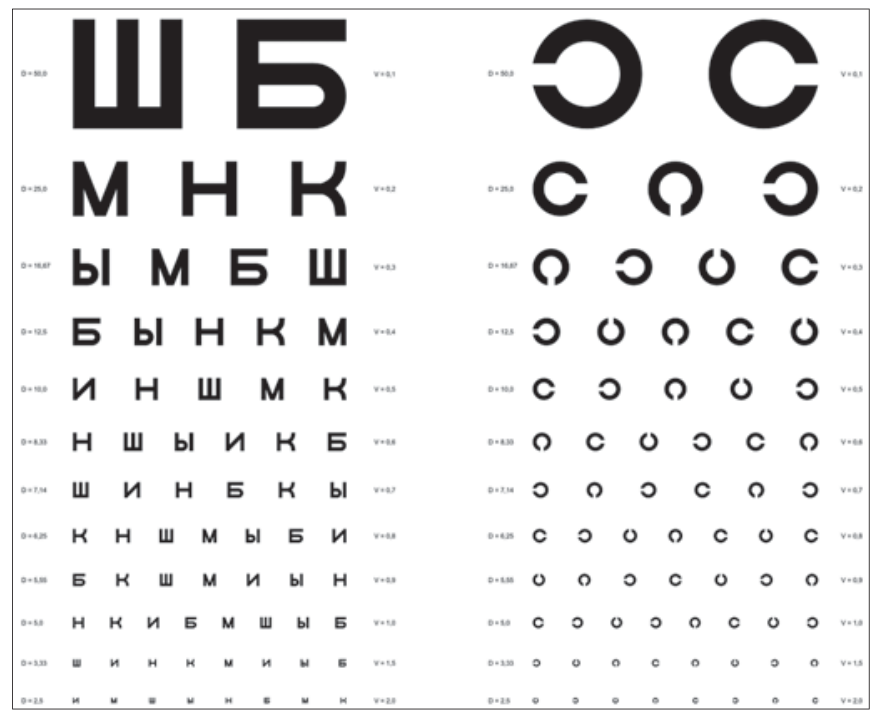

Рис. 2. Таблица Сивцева - Головина

Fig. 2. Sivtzev - Golovin chart

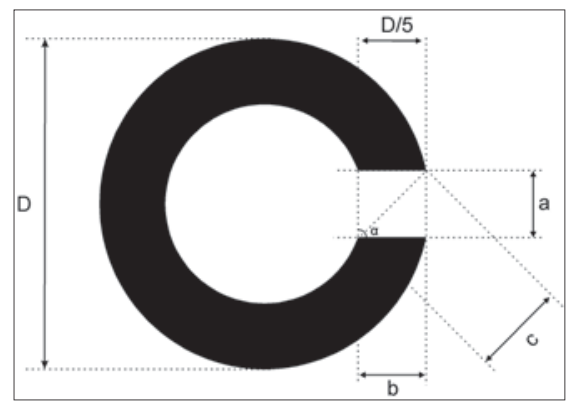

Рис. 3. Кольцо Ландольта

Fig. 3. Landolt ring за счет истончения в области разрыва еще до того, как разрыв становится отчетливо видимым [1].

Вышеизложенное снижает объективность и точность визометрических исследований с использованием оптотипов в виде кольца Ландольта.

Важно также отметить, что значительное влияние на результаты визометрических исследований оказывает принцип построения таблиц для исследования О3.

Многие современные визометрические таблицы построены по десятичной системе, предложенной F. Monoyer [21]. В таблицах, построенных по принципу арифметической прогрессии, каждый ряд букв отличается от соседнего на 0,1 О3. Таблица имеет 10 строк. Цифры О3 расположены на каждой строке с правой стороны в виде десятичной дроби. Определение О3 проводится по данной таблице с расстояния $5 \mathrm{м}$.

Принцип арифметической прогрессии заложен в основу большинства таблиц для визометрии. Однако таблицы, построенные по такому принципу, имеют ряд недостатков. Так, например, в случае О3 от 0,1 до 0,2 происходит увеличение размеров тестобъектов в 2 раза, а при переходе О3 от 0,5 к 0,6 в 1,2 раза. В связи с этим использование данных таблиц значительно снижает чувствительность и точность метода визометрии.

В попытках усовершенствования методов визометрии I. Bailey и J. Lovie [22] предложили таблицы, в которых используется геометрическая прогрессия изменения размеров оптотипов со знаменателем 1,26. В таблицеэтихавторовчисло букв в каждой строке 5, при этом расстояние между краями букв в строке находится в зависимости от ширины букв, а расстояние между краями строк - от высоты букв. Уменьшение размеров букв в каждой последующей строке составляет 26 \%, а через каждые 3 строчки размер оптотипов уменьшается в 2 раза. В данной таблице О3 выражается в непривычных единицах $\log$ MAR (логарифм минимального угла разрешения).

C целью устранения вышеуказанных недостатков нами были предложены оптотип и таблица для визометрических исследований. Суть наших предложений заключается в следующем.

Во-первых, нами разработан новый оптотип, представляющий собой модифицированное кольцо Ландольта. Главной отличительной особенностью предложенного оптотипа является наличие разрыва в виде круга, что позволяет устранить феномен «закругления углов» за счет меридиональной равномерности детали предъявляемого оптотипа (рис. 5).

Оптотип имеет следующую конфигурацию: диаметр $\mathrm{D}$, ширина кольца и диаметр разрыва равны D/5. Так сохраняется оптимальное отношение элементов оптотипа

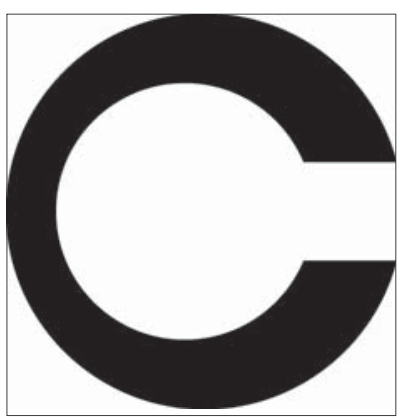
к его общему размеру [4, 15]. Данный оптотип может применяться для визометрических исследований, как в клинической практике, так и для научноисследовательских целей. В частности, наибольшее значение такое строение оптотипа имеет для прецизионных визометрических исследований при астигма-

Рис. 4. Уравновешенное кольцо Вейнберга

Fig. 4. Veinbergs balanced ring 


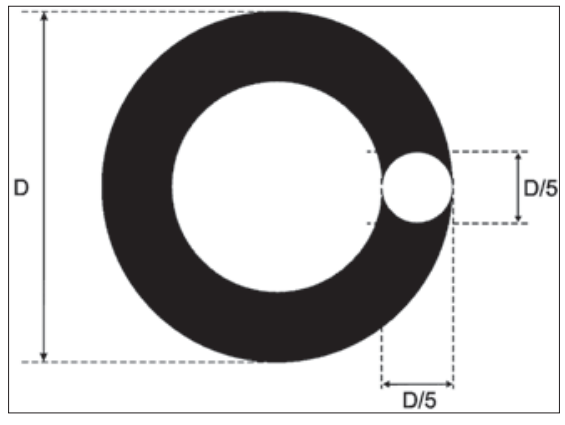

Рис. 5. Новый оптотип для визометрических исследований Fig. 5. New optotype for vision acuity determination

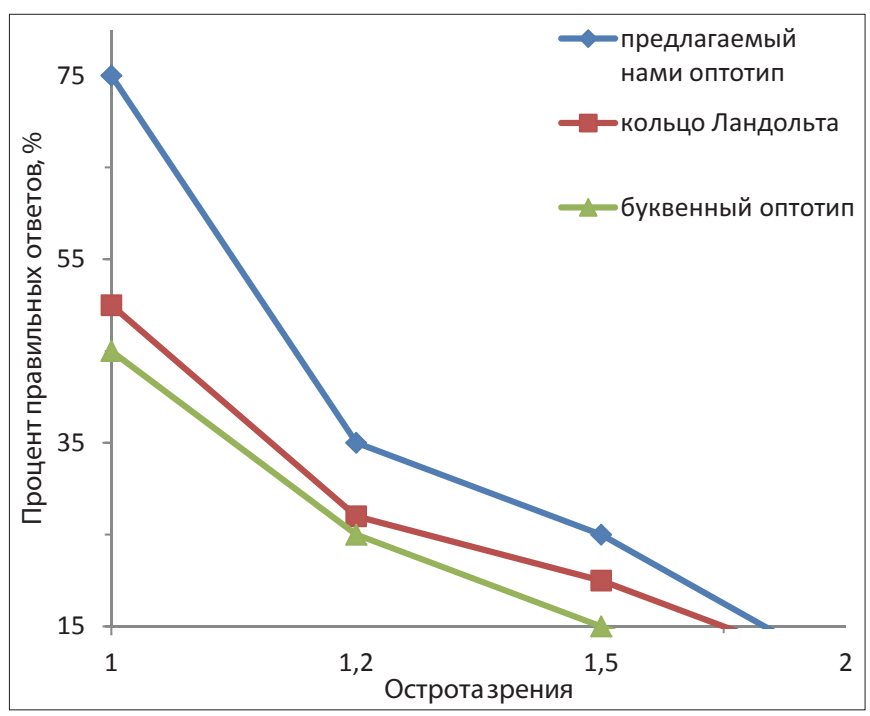

Рис. 7. Сравнительные психометрические кривые распознавания визометрических оптотипов

Fig. 7. Comparative psychometric curves isometrically recognition of the optotypes

тизме, так как в нем соблюдается принцип меридиональной равномерности детали оптотипа.

Во-вторых, нами предлагается таблица (рис. 6), построенная на основе принципа логарифмической прогрессии размеров оптотипов со знаменателем прогрессии 1,25 , т. е. каждая последующая строка в 1,25 раза меньше предыдущей.

Для большей практичности эксплуатации и стандартизации условий освещенности таблица адаптирована для размещения в аппарате Рота. Использование этой таблицы позволяет проводить прецизионные исследования О3 от 0,05 до 2,28 со значительно меньшим шагом, чем стандартная таблица с арифметической прогрессией.

\section{МАТЕРИАЛ И МЕТОДЫ}

Для сравнительной оценки клинической эффективности известных визометрических оптотипов (буквенных, колец Ландольта) с предлагаемым оптотипом нами обследовано 56 пациентов в возрасте от 16 до 57 лет. Критерием включения в исследование явилась максимальная О3 = 1,0 и более.

При отборе пациентов и оценке результатов исследования применялось правило, согласно которому строка таблицы засчитывалась как прочтенная в том случае, если правильно были названы хотя бы 3 оптотипа из 5.

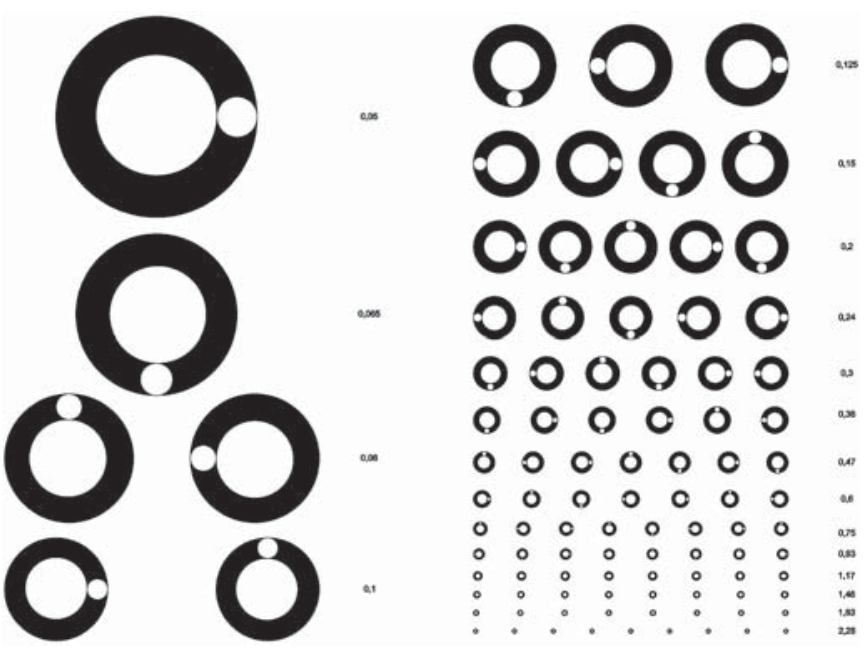

Рис. 6. Модифицированная таблица для визометрических исследований

Fig. 6. Modified chart for vision acuity determination

Всем пациентам исследовали О3 с использованием трех различных оптотипов: стандартных буквенных оптотипов по таблице Сивцева - Головина, колец Ландольта и предлагаемого нами оптотипа.

\section{РЕЗУЛЬТАТЫ И ОБСУЖДЕНИЕ}

На основе регистрации процента правильных ответов нами были построены психометрические кривые для каждого из исследуемых оптотипов (рис. 7), где на оси абсцисс отображается величина О3, а на оси ординат - процент правильных ответов.

Психофизиологическими исследованиями ряда авторов [23-27] было обнаружено, что взаимосвязь между вероятностью наблюдения оптотипа и размером оптотипа обычно описывается кумулятивной функцией распределения вероятности, гладкой синусоидальной функцией, которая является интегралом от нормальной функции распределения. Данную функцию называют психометрической.

Психометрическая кривая при использовании предложенного нами оптотипа превосходит кривые для колец Ландольта и буквенных оптотипов, поскольку он обладает меридиональной изометричностью как в целом, так и в деталях, в то время как кольцо Ландольта в целом является меридионально изометричным, но его деталь (разрыв) остается анизометричной.

Таким образом, предложенный нами оптотип может использоваться для прецизионных визометрических исследований как в клинических, так и в научно-исследовательских целях.

\section{ВЫВОДЫ}

1. Предложены новый оптотип и таблица для визометрических исследований.

2. Использование предложенного оптотипа и таблицы для визометрических исследований, построенной по принципу логарифмической прогрессии размеров оптотипов, позволяет значительно повысить достоверность оценки O3, а расширенный диапазон величин оптотипов повышает чувствительность методики визометрии.

\section{Jumepamypa/References}

1. Волков В.В., Горбань А.Н., Джалиашвили О.А. Клиническая визо- и рефрактометрия. Ленинград: Медицина; 1976. 
Volkov V.V., Gorban' A.N., Dzhaliashvili O.A. Clinical vision and refractometry. Leningrad: Meditsina; 1976 (in Russian).

2. $\quad$ Daza de Valdes B. The use of eye glasses. 1623. In: Runge P.E., ed. History of Ophthalmology. Wayenborgh, Belgium. 2004; 11.

3. Jurin J. An essay on distinct and indistinct vision. In: A complete system of optics in four books, viz. a popular, a mathematical, a mechanical, and a philosophical treatise. Cambridge: Published by the author; 1738: 115-71.

4. Лещенко И.А. О системах и правилах определения остроты зрения. Вестник оптометрии. 2009; 3: 54-8.

Leshchenko I.A. On systems and rules for the determination of visual acuity. Vestnik optometrii. 2009; 3: 54-8 (in Russian).

5. Donders F.C. On the anomalies of accommodation and refraction. London: New Sydenham Society; 1864.

6. Colenbrander $A$. The historical evolution of visual acuity measurement. Vis. Impair. Res. 2008; 10: 57-66. https://doi. org/10.1080/13882350802632401

7. Snellen H. Letterproeven tot Bepaling der Gezictsscherpte. Utrecht, Weyers; 1867 (Dutch edition).

8. Воронцов Е.A., Черноусов А.С. Классификация методов и средств определения остроты зрения. Научно-технический вестник информационных технологий, механики и оптики 2006; 28: 75-83.

Vorontsov E.A., Chernousov A.S. Classification of methods and means for determining visual acuity. Nauchno-tekhnicheskiy vestnik informatsionnykh tekhnologiy, mekhaniki i optiki. 2006; 28: 75-83 (in Russian).

9. Landolt E. Bestimmung der Sehschärfe. In: Die Functionsprüfungen des Auges. Graefe-Saemisch Handbuch, 2nd edition; Leipzig; 1904.

10. Bennett A.G. Ophthalmic test types. Br.J. Physiol. Optics.1965;22:238-71.

11. Sloan L.L. New test charts for the measurement of visual acuity at far and near distances. Am. J. Ophthalmol. 1959; 48: 807-13.

12. Ferree $C$., Rand $G$. More nearly absolute method of testing and rating vision. Arch. Ophthalmol.1940; 24 (2): 292-315.

13. Jonkers G.H. A new optotype chart. Ophthalmologica. 1975; 171: 380-1. https://doi.org/10.1159/000307553

14. Ferris F.L., Kassov A., Bresnick G.H., Bailey I.L. New visual acuity charts for clinical research. Am. J. Ophthalmol. 1982; 94: 91-6.

15. Taylor H.R. Applying new design principles to the construction of an Illiterate E chart. Am. J. Optom. Physiol. Optics.1978; 55: $348-51$.

16. Ferree $C$., Rand $G$. The testing of visual acuity. Factors in the sensitive use of the test for the detection of errors of refraction. Am. J. Optom. 1934; 17 (1 Jan.): 29-36. https://doi.org/10.1016/ S0002-9394(34)92090-0

Поступила: 27.05.2019

Переработана: 19.07.2019

Принята к печати: 27.08.2019
17. Вейнберг В.Б., Никольская Н.А. Таблицы для измерения остроты зрения. Проблемы физиологической оптики, изд. 8. Москва: Издательство АН СССР; 1951: 329-52.

Vejnberg V.B., Nikol'skaja N.A. Tables for determining visual acuity. Problemy fiziologicheskoj optiki, izd. 8. Moscow: Izdatel'stvo AN SSSR; 1951: 329-52 (in Russian).

18. Westheimer $G$. Optotype recognition under degradation: comparison of size, contrast, blur, noise and contour perturbation effects. Clin. Exp. Optom. 2016; 99: 66-72. doi: 10.1111/ cхо. 12293

19. Алиев А-Г.Д., Исмаилов М.И. Исследование феномена псевдоаккомодации при интраокулярной коррекции афакии. Офтальмохирургия. 1999; 4: 38-42.

Aliev A-G.D., Ismailov M.I. Investigation of the phenomenon of pseudo-accommodation for intraocular correction of aphakia. Oftal'mokhirurgiya. 1999; 4: 38-42 (in Russian).

20. Sergienko N.M., Aliyev A-H.D. Correcting astigmatism. Optom. Vis. Sci. 1989; 66 (3): 167-9.

21. Monoyer F. Echelle typographique décimale pour mesurer l'acuit visuelle. Gaz. Med. Paris.1875; 21: 258.

22. Bailey I.L., Lovie J.E. New design principles for visual acuity letter charts. Am. J. Optom. Physiol. Opt.1976; 53: 740.

23. Проскурина О.В., Розенблюм Ю.З., Берианский М.И. Таблица для исследования остроты зрения у детей. Вестник офтальмологии. 1998; 3: 43-5.

Proskurina O.V., Rozenblum Yu.Z., Bershanskiy M.I. Chart for the study of visual acuity in children. Vestnik oftal'mologii. 1998; 3: 43-5 (in Russian).

24. Alexander K., Xie W., Derlacki D. Visual acuity and contrast sensitivity for individual sloan letters. Vision Res. 1997; 37 (6 Mar.): 813-9. https://doi.org/10.1016/S0042-6989(96) 00190-3

25. Tokutake T., Mita N., Kawamoto K.-I., et al. Relation between visual acuity and slope of psychometric function in young adults. i-Perception. 2011; 2: 308-8. doi: 10.1068/ic308

26. Simpson $T$. Vision thresholds from psychometric analyses: alternatives to Probit analysis. Optom. Vis. Sci. 1995; 72: 371-7. doi:10.1097/00006324-199506000-00004

27. Schulze-Bonsel K., Feltgen N., Burau H., Hansen L., Bach M. Visual acuities "hand motion" and "counting fingers" can be quantified with the freiburg visual acuity test. Invest. Ophthalmol. Vis. Sci. 2006; 47 (3 Mar.): 1236-40. doi:10.1167/iovs.05-0981

Originally received: 27.05 .2019

Final revision: 19.07.2019

Accepted: 27.08.2019

\section{ИНФОРМАЦИЯ ОБ АВТОРАХ/INFORMATION ABOUT THE AUTHORS}

ГБОУ ВПО «Дагестанский государственный медицинский университет», пл. Ленина, д. 1, г. Махачкала, 367000, Российская Федерация, Республика Дагестан Абдул-Гамид Давудович Алиев, д-р мед. наук, профессор, заведующий кафедрой глазных болезней № 1 ГБУНКО «Дагестанский центр микрохирургии глаза», ул. Набережная, д. 12, г. Каспийск, 368300, Российская Федерация, Республика Дагестан

Ахмед Абдул-Гамидович Алиев, канд. мед. наук, заместитель директора по научно-исследовательской работе

Муса Муртазалиевич Нурудинов, врач-офтальмолог отделения новых технологий и витреоретинальной хирургии

Для контактов: Муса Муртазалиевич Нурудинов, musail1988@gmail.com
Chair of ophthalmology, Daghestan State Medical University, 1, Ploshchad Lenina, Makhachkala, 367000, Russia

Abdul-Gamid D. Aliev, Dr. of Med. Sci., Professor, Head of Chair of Ophthalmology № 1

Daghestan Center of Eye microsurgery, 12, Naberezhnaya St., Kaspiysk, 368300, Russia

Akhmed A.-G. Aliev, Cand. of Med. Sci., deputy director for science

Musa M. Nurudinov, ophthalmologist, department of vitreoretinal surgery and new technologies

Contact information: Musa M. Nurudinov, musail1988@gmail.com 